Check for updates

Cite this: RSC Adv., 2017, 7, 44333

Received 2nd September 2017

Accepted 8th September 2017

DOI: 10.1039/c7ra09770k

rsc.li/rsc-advances

\section{Annulation of 2,3-diphenyl-4H-chromen-4-ones via photo-induced hydrogen evolution $\uparrow$}

\author{
Yang Kang, (D) a Tao Wang, (D) a Yong Liang, ${ }^{b}$ Yangyang Zhang, ${ }^{a}$ Rui Wang ${ }^{a}$ \\ and Zunting Zhang (D) *a
}

An efficient photo-induced transition-metal-free direct hydrogen evolution and annulation of 2,3diphenyl-4H-chromen-4-ones in $\mathrm{EtOH}-\mathrm{H}_{2} \mathrm{O}(19: 1, \mathrm{v} / \mathrm{v})$ at room temperature was described. The reported methodology provided a quick and easy access to the synthesis of dibenzo[a,c]xanthen derivatives, which eliminates the use of any catalysts, oxidants and additives.

\section{Introduction}

In recent years, photochemical reactions have drawn great attention due to their environmentally friendly properties, sustainability and high atom efficiency compared to conventional transition-metal catalyzed reactions. ${ }^{1,2}$ Furthermore, photons have been recognized as an ideal clean reagent for organic reactions. ${ }^{3,4}$ Photochemical reactions have been successfully applied to the synthesis of phenanthrene derivatives and polycyclic aromatic hydrocarbons (PAHs), ${ }^{5,6}$ which are difficult to achieve via traditional chemical conditions.

Chromones are the core structures of flavonoids and isoflavonoids and have been extensively studied as biologically active substrates as well as drug molecules. ${ }^{7,8}$ Owing to their tremendous significance in biological and pharmaceutical activities, ${ }^{9}$ great effort has been made in the development of various chromone derivatives. Recently, transition-metal catalyzed $\mathrm{C}-\mathrm{H}$ activation and annulation have been successfully applied for the synthesis of polycyclic derivatives of chromones. Yoshikai et al. reported $\operatorname{Pd}(\mathrm{dba})_{2}$ catalyzed cyclization of 3iodoflavones with 2-(trimethylsilyl)phenyl triflate in the presence of $\mathrm{CsF}$ and $\mathrm{P}(o \text {-tolyl })_{3}$ at $110^{\circ} \mathrm{C}$ for $24 \mathrm{~h}$ to give dibenzo[a,c] xanthen derivative $2 \mathrm{a}$ in $79 \%$ (Scheme 1a). ${ }^{10}$

Alternatively, the irradiation of 2,3-diphenyl-4H-chromen-4one 1a in acetone with a medium pressure mercury lamp under the air atmosphere for $1 \mathrm{~h}$ yielded trace amount of $\mathbf{2 a}$ $(5 \%$, Scheme $1 \mathrm{~b}) .{ }^{11}$ It was proposed that $2 \mathrm{a}$ was generated via the well-known stilbene-phenanthrene type photocyclization of 2,3-diphenyl-4H-chromen-4-one 1a. Generally speaking,

${ }^{a}$ Key Laboratory of the Ministry of Education for Medicinal Resources and Natural Pharmaceutical Chemistry, National Engineering Laboratory for Resource Development of Endangered Crude Drugs in Northwest of China, School of Chemistry and Chemical Engineering, Shaanxi Normal University, Xi'an 710119, People's Republic of China. E-mail: zhangzunting@sina.com

${ }^{b}$ Department of Molecular Medicine, Beckman Research Institute, Hope Duarte, CA 91010, USA

$\dagger$ Electronic supplementary information (ESI) available. See DOI: 10.1039/c7ra09770k photocyclization of stilbene analogues always require the presence of oxidant $\left(\mathrm{O}_{2}\right.$ or $\mathrm{I}_{2}, \mathrm{KI}, \mathrm{CuCl}_{2}, \mathrm{TCNE}$ et al. $)$ for the reaction to proceed smoothly. ${ }^{12-14}$ Luckily, we were able to obtain the cyclization products of 2,3-di(hetero)aryl-4H-chromen-4-ones without the requirement of any oxidant or additives. ${ }^{15}$ However, the annulation product for less reactive 2,3-diphenyl$4 H$-chromen-4-one substrate was not obtained. Following our investigation in the development of photo-induced transition metal-free cross-coupling reaction ${ }^{16}$ as well as the direct oxidative annulation, ${ }^{15,17,18}$ we would like to extend an efficient photoinduced intramolecular hydrogen evolution and annulation to 2,3-diphenyl-4H-chromen-4-ones substrate, which provides access to dibenzo[ $[a, c]$ xanthen analogues (Scheme 1c).

\section{Results and discussion}

Initially, the photo-induced oxidative annulation of 2,3diphenyl-4H-chromen-4-one (1a) was optimized and the

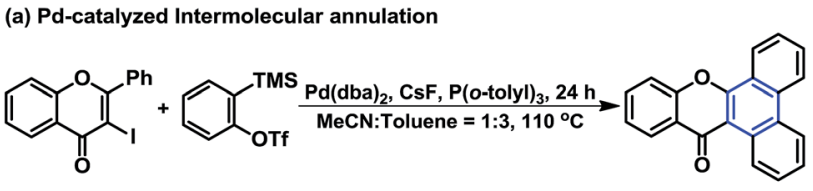

(b) Oxidative annulation
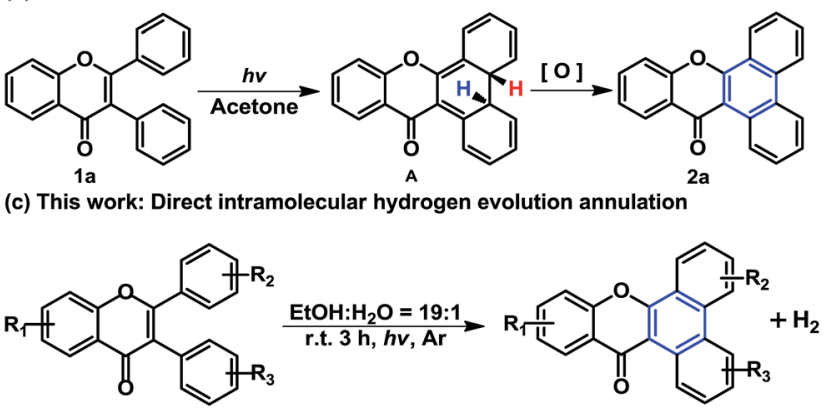

$\mathbf{R}^{1}=\mathrm{H}, \mathrm{OMe}, \mathrm{Me}, \mathrm{F}, \mathrm{OH} ; \mathbf{R}^{3}=\mathrm{H}, \mathrm{Me}, \mathrm{t}-\mathrm{Bu} \mathrm{Cl}, \mathrm{F} ; \mathbf{R}^{2}=\mathrm{H}, \mathrm{OMe}, \mathrm{F}, \mathrm{Me}, \mathrm{OH}$.

Scheme 1 Synthetic approaches to dibenzo[a,c]xanthenes. 
corresponding data were presented in Table 1 . Thus, irradiation of $1 \mathrm{a}$ in acetonitrile $(100 \mathrm{~mL})$ with a $500 \mathrm{~W}$ high-pressure mercury lamp at ambient temperature under argon atmosphere for $3 \mathrm{~h}$ gave $2 \mathrm{a}$ in the yield of $49 \%$ (entry 1). Annulation of 1a in acetone or dichloromethane did not improve the yield of $2 \mathbf{a}$ (38-43\%, entries $2-3)$. While, similar yield of 2 a was obtained in ethanol (56\%, entry 4$)$. It was interesting to find out that the yields of 2a were significantly affected with the presence of $\mathrm{H}_{2} \mathrm{O}$ (entries 5-8). The amount of additional water played a critical role towards the cyclization, since $\mathbf{2 a}$ was obtained in lower yields with either too much or too little water. The optimal yield was obtained when $\mathrm{EtOH} / \mathrm{H}_{2} \mathrm{O}(19: 1, \mathrm{v} / \mathrm{v})$ was chosen as solvent (63\%, entry 7$)$. Meanwhile, the concentration of 1a was also explored (entries 7, 9-10) and 2a was obtained in highest yield when the reaction was performed in $5 \mathrm{mM}$ scale $(63 \%$, entry 7). Finally, screening of irradiation time was conducted and lower yield of 2 a was obtained (entries 11-12). When the cyclization was performed under open air, 2a was obtained in $32 \%$ yield (entry 13). Thus, the irradiation of $5 \mathrm{mM} 1 \mathrm{a}$ in EtOH$\mathrm{H}_{2} \mathrm{O}(19: 1, \mathrm{v} / \mathrm{v})$ at ambient temperature for $3 \mathrm{~h}$ under the argon atmosphere was determined to be the optimal condition.

With the optimized conditions in hand, the tolerance of various functional groups (methyl, tert-butyl, fluoro, chloro, hydroxyl and methoxyl) has been explored and the yields were summarized in Table 2. Generally speaking, the substrates bearing electron-donating groups (e.g., $\mathrm{Me}, \mathrm{OMe}, t$-Bu) gave the corresponding products $\mathbf{2 a - 2 p}$ in higher good yields (59-81\%) comparing to those (2q-2x, 54-69\%) bearing electronwithdrawing groups (e.g., F, Cl). Notably, the substrates bearing free hydroxyl group also yielded the corresponding annulation products $2 \mathbf{y}$ and $\mathbf{2 z}$ in high yields, which are better than the yields of $\mathbf{2 l}$ and $\mathbf{2 e}$, respectively. There is a significant difference in the irradiation time as well. Substrates containing EWG usually require longer reaction time than those with EDG. The structures of 2 were characterized by ${ }^{1} \mathrm{H}$ NMR, ${ }^{13} \mathrm{C}$ NMR, HRMS and IR.

Based on our experimental data and a literature report, ${ }^{19}$ a plausible mechanism for the formation of $\mathbf{2 a}$ is depicted in Scheme 2. Initially, the irritation of 2,3-diphenyl-4H-chromen4-one (1a) with a high-pressure mercury lamp generate intermediate A, followed by a thermal suprafacial $[1,5]-\mathrm{H}$ shift ${ }^{20}$ to give intermediate $\mathrm{B}$. Spontaneous rearomatization of benzene ring is believed to be the force driving for [1,5]-sigmatropic shift. Subsequently, keto-enol isomerization of B led to the formation of a more stable syn-isomer C. Similar transformations have been reported for a number of structurally related compounds. ${ }^{\mathbf{1 5 1 - 2 4}}$ It is worth mentioning that polar protic solvent EtOH- $\mathrm{H}_{2} \mathrm{O}(19: 1, \mathrm{v} / \mathrm{v})$ plays an important role in the process of keto-enol isomerization, which accounts for higher yields of 2 a comparing to $\mathrm{MeCN}, \mathrm{CHCl}_{2}$ and $\mathrm{Me}_{2} \mathrm{CO}$. In the end, the annulation product $2 \mathrm{a}$ was obtained along with the elimination of hydrogen molecule via syn-elimination from intermediate $\mathrm{C}$, which could be easily explained by the restoration of aromaticity for the benzene ring and the entire conjugated system. To our delight, we have successfully detected the hydrogen via GC and the detail experiment was shown in ESI-Fig. 2. $\dagger$ The annulation product 2a was also produced under the open air (oxygen, Table 1, entry 13), and it indicated that the photochemical cyclization proceed through the S1 state.

Encouraged by the further certified the proposed mechanism, we turned our attention to the reductive carbonyl. 1a was reduced to 3 under the presence of $\mathrm{LiAlH}_{4} \mathrm{AlCl}_{3}$ in THF at $0{ }^{\circ} \mathrm{C}$ for $30 \mathrm{~min}$ to gave 3 (yield $44 \%$, Scheme 3 ). ${ }^{25}$ As expected, no

Table 1 Optimization for the annulation of $1 a^{a}$

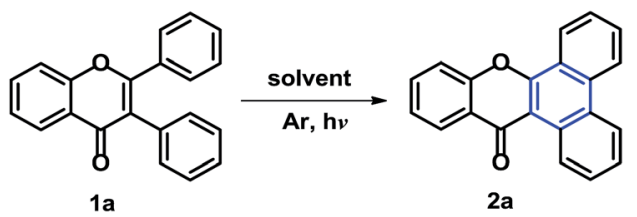

\begin{tabular}{|c|c|c|c|c|c|}
\hline Entry & Concn (mM) & Solvent (v/v) & Time (h) & $\operatorname{Conv}^{b}(\%)$ & Yield $^{b}(\%)$ \\
\hline 1 & 5 & $\mathrm{MeCN}$ & 3 & 73 & 49 \\
\hline 2 & 5 & $\mathrm{Me}_{2} \mathrm{CO}$ & 3 & 69 & 38 \\
\hline 4 & 5 & EtOH & 3 & 75 & 56 \\
\hline 5 & 5 & EtOH- $\mathrm{H}_{2} \mathrm{O}(7: 1)$ & 3 & 83 & 38 \\
\hline 6 & 5 & $\mathrm{EtOH}-\mathrm{H}_{2} \mathrm{O}(13: 1)$ & 3 & 72 & 45 \\
\hline 9 & 3 & EtOH- $\mathrm{H}_{2} \mathrm{O}(19: 1)$ & 3 & 96 & 37 \\
\hline 10 & 7 & $\mathrm{EtOH}-\mathrm{H}_{2} \mathrm{O}(19: 1)$ & 3 & 70 & 41 \\
\hline 11 & 5 & $\mathrm{EtOH}-\mathrm{H}_{2} \mathrm{O}(19: 1)$ & 5 & 100 & 51 \\
\hline 12 & 5 & $\mathrm{EtOH}-\mathrm{H}_{2} \mathrm{O}(19: 1)$ & 1 & 54 & 36 \\
\hline 13 & 5 & $\mathrm{EtOH}-\mathrm{H}_{2} \mathrm{O}(19: 1)$ & 3 & 79 & $32^{c}$ \\
\hline
\end{tabular}

${ }^{a} 0.5 \mathrm{mmol} 1 \mathrm{a}$ in various solvent $(100 \mathrm{~mL}, 5 \mathrm{mM})$ was irradiated with a $500 \mathrm{~W}$ high-pressure mercury lamp at room temperature in the argon atmosphere. ${ }^{b}$ Isolated yield. ${ }^{c}$ In the open air atmosphere. 


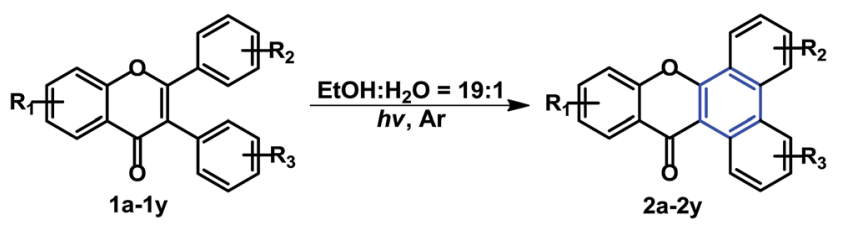<smiles>O=c1c2ccccc2oc2c3ccccc3c3ccccc3c12</smiles>

2a, $63 \%, 3 \mathrm{~h}$<smiles>Cc1ccc2oc3c4ccccc4c4ccccc4c3c(=O)c2c1</smiles>

2e, $62 \%$, 3h<smiles>Cc1ccc2c(c1)-c1ccc(C)cc3c(c4ccc(C)cc4c(=O)c1-3)O2</smiles>

2i, $72 \%$, 3h<smiles>Cc1ccc2c(c1)c1ccccc1c1c(=O)c3ccccc3oc21</smiles>

2m, 75\%, 3h<smiles>Cc1ccc2c(c1)c1cc(F)ccc1c1c(=O)c3ccccc3oc21</smiles>

2q, $55 \%, 5.5 \mathrm{~h}$<smiles>Cc1ccc2c(c1)c1ccccc1c1c(=O)c3cc(F)ccc3oc21</smiles>

$2 \mathrm{u},{ }^{b} 54 \%, 5 \mathrm{~h}$<smiles>Cc1ccc2c(c1)c1cc(C(C)C)ccc1c1c(=O)c3ccc(O)cc3oc21</smiles>

$2 y,{ }^{b} 78 \%, 3 \mathrm{~h}$<smiles>COc1ccc2c(c1)c1ccccc1c1c(=O)c3cc(C)ccc3oc21</smiles>

2b, $72 \%, 3 \mathrm{~h}$<smiles>Cc1ccc2oc3c4ccccc4c4cc(CC(C)C)ccc4c3c(=O)c2c1</smiles>

2f, $71 \%$, $3 \mathrm{~h}$<smiles>Cc1ccc2c(c1)-c1cc(CC(C)C)ccc3c(c4ccc(C)cc4c(=O)c1-3)O2</smiles>

2j, $78 \%, 3 \mathrm{~h}$<smiles>COc1ccc2c(=O)c3c4ccccc4c4cc(C)ccc4c3oc2c1</smiles>

2n, $59 \%, 3 \mathrm{~h}$<smiles>COc1ccc2c(=O)c3c4ccc(Cl)cc4c4cc(C)ccc4c3oc2c1</smiles>

$2 \mathbf{r}^{b} 58 \%, 4.5 \mathrm{~h}$<smiles>Cc1ccc2c(c1)c1cc(C(C)(C)C)ccc1c1c(=O)c3cc(F)ccc3oc21</smiles>

$2 v,{ }^{b} 63 \%, 4.5 \mathrm{~h}$<smiles>Cc1ccc2oc3c4ccc(O)cc4c4ccccc4c3c(=O)c2c1</smiles>

2z, ${ }^{b} 70 \%, 3 \mathrm{~h}$<smiles></smiles>

2d, $81 \%, 3 \mathrm{~h}$<smiles>Cc1ccc2c(=O)c3c4cc(C)ccc4oc3c3ccccc3c2c1</smiles>

2g, $68 \%, 3 \mathrm{~h}$<smiles>Cc1ccc2c(c1)c1ccccc1c1c(=O)c3ccccc3oc21</smiles>

2k, $70 \%, 3$ h<smiles>COc1ccc2c(=O)c3c4cc(C)ccc4c4cc(C)ccc4c3oc2c1</smiles>

2o, $70 \%$, 3h<smiles>COc1ccc2c(c1)c1cc(F)ccc1c1c(=O)c3cc(C)ccc3oc21</smiles>

$2 \mathbf{s}^{b} 64 \%, 5 \mathrm{~h}$<smiles></smiles>

2h, $74 \%$, 3h<smiles>Cc1ccc2c(c1)c1cc(CC(C)C)ccc1c1c(=O)c3ccccc3oc21</smiles>

2I, 73\%, 3h<smiles>COc1ccc2c(=O)c3c4ccc(CC(C)C)cc4c4cc(C)ccc4c3oc2c1</smiles>

2p, $71 \%$, 3h<smiles>COc1ccc2c(=O)c3c4ccc(F)cc4c4cc(C)ccc4c3oc2c1</smiles>

$2 t^{b}, 55 \%, 5.5 \mathrm{~h}$<smiles></smiles>

$2 x^{b}, 69 \%, 5.5 h$

${ }^{a}$ All reactions were carried out on the $0.5 \mathrm{mmol}$ scale with the irradiation of $500 \mathrm{~W}$ high-pressure mercury lamp under the argon atmosphere at room temperature until substrate was completely consumed (TLC monitor). ${ }^{b}$ The yields of $2 \mathbf{q}-2 \mathbf{x}$ was $39 \%, 45 \%, 49 \%, 31 \%, 46 \%, 40 \%, 43 \%$, and $55 \%$, for $3 \mathrm{~h}$, respectively.

annulation product $\mathbf{4}$ was detected at the optimal reaction conditions, which could be explained to the lack of keto-enol tautomerization.

\section{Conclusions}

In summary, we have demonstrated a simple photo-induced direct hydrogen evolution and annulation for the synthesis of 
<smiles></smiles>

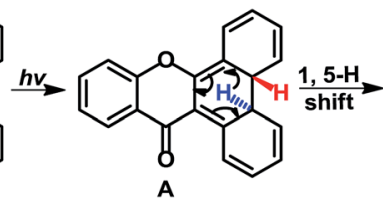<smiles>C[C@]12C=CC=C[C@@H]1c1ccccc1-c1oc3ccccc3c(=O)c12</smiles>
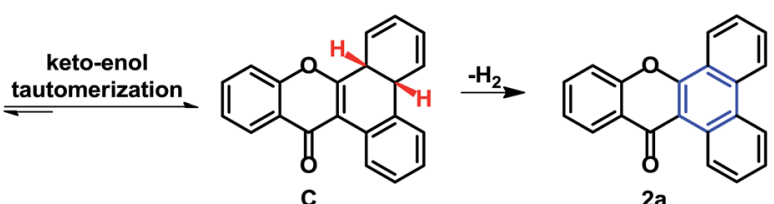

Scheme 2 Proposed reaction mechanism for the formation of $2 a$

14H-dibenzo[ $a, c]$ xanthen-14-one derivatives in 95\% EtOH under room temperature. Comparing to conventional transitionmetal-catalyzed annulation and oxidative photo-cyclization reaction, the reported methodology offers several notable advantages, including simple operation, mild reaction condition, no catalysts, oxidants and additives. Meanwhile, the failure of the cyclization of 3 and detection the $\mathrm{H}_{2}$ generated by the annulation of $1 \mathrm{n}$ sufficiently proved the rationality of the proposed mechanism.

\section{Experimental sections}

Unless otherwise noted, commercial reagents were purchased from Energy Chemical. All experiments were determined by thin layer chromatography (TLC). TLC used silica gel 60 GF254 plate. Column chromatography (200-300 mesh) was performed on silica gel. ${ }^{1} \mathrm{H}$ and ${ }^{13} \mathrm{C}$ NMR spectra were recorded on Bruker 400 or $600 \mathrm{MHzspectrometer.}{ }^{1} \mathrm{H}$ and ${ }^{13} \mathrm{C}$ NMR spectra were reported in parts per million ( $\mathrm{ppm}$ ) and referenced to the residual solvent peaks $\left[\mathrm{CDCl}_{3}(7.26 \mathrm{ppm})\right.$ or DMSO- $\left.d_{6}(2.50)\right]$ for ${ }^{1} \mathrm{H}$ NMR and the $\mathrm{CDCl}_{3}$ (77.16 ppm) or DMSO- $d_{6}(39.52)$ for ${ }^{13} \mathrm{C}$ NMR spectra. High-resolution mass spectra (HRMS) were obtained using the electron-spray ionization (ESI) technique. Melting points were measured with a X-5 micro-melting point apparatus and were uncorrected. IR spectra were recorded with a Nicollet 170SX FT-IR spectrophotometer with $\mathrm{KBr}$ pellets. According to the literature reported for the synthesis of 3-iodo2-phenyl-4H-chromen-4-one, which using 2-phenyl-4Hchromen-4-ones $(1 \mathrm{mmol}), \mathrm{I}_{2}(304 \mathrm{mg}, 1.2 \mathrm{mmol})$ and $\mathrm{Ce}\left(\mathrm{NH}_{4}\right)_{2}\left(\mathrm{NO}_{3}\right)_{6}(603 \mathrm{mg}, 1.1 \mathrm{mmol})$ as starting materials and pre-dried MeCN $(10 \mathrm{~mL})$ as solvent a series of 3-iodoflavonoids compounds was obtained. ${ }^{26}$
General procedure for the synthesis of compounds $2 \mathrm{a}-2 \mathrm{z}$

Substrate 2,3-diphenyl-4H-chromen-4-one 1a (149 mg, 0.5 $\mathrm{mmol})$ was dissolved in $\mathrm{EtOH}-\mathrm{H}_{2} \mathrm{O}(100 \mathrm{~mL}, 19: 1, \mathrm{v} / \mathrm{v})$ at ambient temperature in a quartz tube, and was degassed for 5 min via ultrasound, deaerated for $20 \mathrm{~min}$ by bubbling argon and irradiated with a high pressure mercury lamp $(500 \mathrm{~W})$ at room temperature for $3 \mathrm{~h}$. Then the reaction mixture were removed under reduced pressure and purified by column chromatography (ethyl acetate/petroleum ether, $1: 50$ ) to give 2a $(63 \%)$.

14H-dibenzo[a,c $]$ xanthen-14-one (2a).$^{10}{ }^{1} \mathrm{H}$ NMR $(400 \mathrm{MHz}$, $\left.\mathrm{CDCl}_{3}\right) \delta 10.19(\mathrm{~d}, J=8.4 \mathrm{~Hz}, 1 \mathrm{H}), 8.77(\mathrm{~d}, J=8.2 \mathrm{~Hz}, 1 \mathrm{H}), 8.68(\mathrm{t}$, $J=8.5 \mathrm{~Hz}, 2 \mathrm{H}), 8.46(\mathrm{~d}, J=7.9 \mathrm{~Hz}, 1 \mathrm{H}), 7.87-7.68(\mathrm{~m}, 6 \mathrm{H}), 7.51-$ $7.47(\mathrm{~m}, 1 \mathrm{H}) ;{ }^{13} \mathrm{C} \mathrm{NMR}\left(100 \mathrm{MHz}, \mathrm{CDCl}_{3}\right) \delta 178.33,155.21$, 154.42, 133.92, 133.80, 130.62, 128.99, 128.57, 127.75, 127.50, $127.32,126.71,126.68,124.71,124.10,123.99,123.97,122.90$, $122.32,117.50,112.70$.

6-methoxy-12-methyl-14H-dibenzo[a,c]xanthen-14-one (2b). White solid. Yield: $72 \%, M_{\mathrm{p}}$ : 229.3-231.9 ${ }^{\circ} \mathrm{C} .{ }^{1} \mathrm{H}$ NMR $(400 \mathrm{MHz}$, $\left.\mathrm{CDCl}_{3}\right) \delta 10.11(\mathrm{~d}, J=8.4 \mathrm{~Hz}, 1 \mathrm{H}), 8.42(\mathrm{dd}, J=8.4,5.7 \mathrm{~Hz}, 2 \mathrm{H})$, $8.14(\mathrm{~s}, 1 \mathrm{H}), 7.82(\mathrm{~d}, J=2.3 \mathrm{~Hz}, 1 \mathrm{H}), 7.69(\mathrm{dd}, J=11.3,4.1 \mathrm{~Hz}$, $1 \mathrm{H}), 7.63-7.53(\mathrm{~m}, 1 \mathrm{H}), 7.48(\mathrm{dd}, J=8.5,2.0 \mathrm{~Hz}, 1 \mathrm{H}), 7.41(\mathrm{~d}, J=$ $8.4 \mathrm{~Hz}, 1 \mathrm{H}), 7.17$ (dd, $J=9.0,2.4 \mathrm{~Hz}, 1 \mathrm{H}), 3.97$ (s, 3H), 2.49 (s, $3 \mathrm{H}) ;{ }^{13} \mathrm{C} \mathrm{NMR}\left(100 \mathrm{MHz}, \mathrm{CDCl}_{3}\right) \delta 178.10,161.54,155.33$, 152.54, 135.81, 134.79, 134.36, 129.75, 128.62, 127.84, 126.85, 126.22, 125.96, 123.59, 122.27, 117.97, 117.18, 116.91, 110.89, 104.35, 55.57, 21.18; IR (KBr), $\nu\left(\mathrm{cm}^{-1}\right)$ 2927, 1610, 1446, 1247, 1029, 925, 821, 628, 530; HRMS (ESI): calc. for $\mathrm{C}_{23} \mathrm{H}_{16} \mathrm{O}_{3}[\mathrm{M}+\mathrm{H}]^{+}$ 341.1178, found 341.1177 .

6-Methoxy-2,12-dimethyl-14H-dibenzo[a,c]xanthen-14-one (2c). White solid. Yield: $78 \%, M_{\mathrm{p}}: 222.4-224.2{ }^{\circ} \mathrm{C}$. ${ }^{1} \mathrm{H}$ NMR $(400 \mathrm{MHz}$, $\left.\mathrm{CDCl}_{3}\right) \delta 9.93(\mathrm{~s}, 1 \mathrm{H}), 8.45(\mathrm{~d}, J=8.4 \mathrm{~Hz}, 1 \mathrm{H}), 8.31(\mathrm{~d}, J=8.4 \mathrm{~Hz}$, $1 \mathrm{H}), 8.15(\mathrm{~s}, 1 \mathrm{H}), 7.81(\mathrm{~s}, 1 \mathrm{H}), 7.50-7.38(\mathrm{~m}, 3 \mathrm{H}), 7.17(\mathrm{~d}, J=9.0 \mathrm{~Hz}$, 1H), $3.99(\mathrm{~s}, 3 \mathrm{H}), 2.60(\mathrm{~s}, 3 \mathrm{H}), 2.50(\mathrm{~s}, 3 \mathrm{H}) ;{ }^{13} \mathrm{C}$ NMR $(100 \mathrm{MHz}$, $\left.\mathrm{CDCl}_{3}\right) \delta 178.20,161.59,155.54,152.61,138.68,135.97,134.74$, 134.32 , 129.82, 127.75, 127.63, 125.98, 124.71, 123.67, 122.20, $117.65,117.20,116.57,110.83,104.14,55.57,22.22,21.21$; IR (KBr), $\nu\left(\mathrm{cm}^{-1}\right)$ 3101, 2914, 1612, 1442, 1238, 1130, 1029, 827, 622, 495; HRMS (ESI): calc. for $\mathrm{C}_{24} \mathrm{H}_{18} \mathrm{O}_{3}[\mathrm{M}+\mathrm{H}]^{+} 355.1334$, found 355.1336.

3-(tert-Butyl)-6-methoxy-12-methyl-14H-dibenzo[a,c]xanthen14-one (2d). Yellow solid. Yield: $81 \%, M_{\mathrm{p}}$ : 255.6-258.1 ${ }^{\circ} \mathrm{C} .{ }^{1} \mathrm{H}$ NMR (400 MHz, $\left.\mathrm{CDCl}_{3}\right) \delta 10.08(\mathrm{~d}, J=8.9 \mathrm{~Hz}, 1 \mathrm{H}), 8.60$ (d, $J=$ $8.9 \mathrm{~Hz}, 1 \mathrm{H}), 8.51(\mathrm{~d}, J=2.3 \mathrm{~Hz}, 1 \mathrm{H}), 8.20$ (s, $1 \mathrm{H}), 8.01$ (d, $J=$ $2.3 \mathrm{~Hz}, 1 \mathrm{H}), 7.83(\mathrm{dd}, J=8.9,2.0 \mathrm{~Hz}, 1 \mathrm{H}), 7.51(\mathrm{~d}, J=2.8 \mathrm{~Hz}, 2 \mathrm{H})$, 7.28 (dd, $J=9.1,2.4 \mathrm{~Hz}, 1 \mathrm{H}), 4.05$ (s, 3H), 2.50 (s, 3H), 1.51 (s, $9 \mathrm{H}) ;{ }^{13} \mathrm{C} \mathrm{NMR}\left(100 \mathrm{MHz}, \mathrm{CDCl}_{3}\right) \delta 178.21,161.58,155.19,152.77$, 149.03, 136.20, 134.80, 134.37, 127.69, 127.53, 126.93, 126.74,

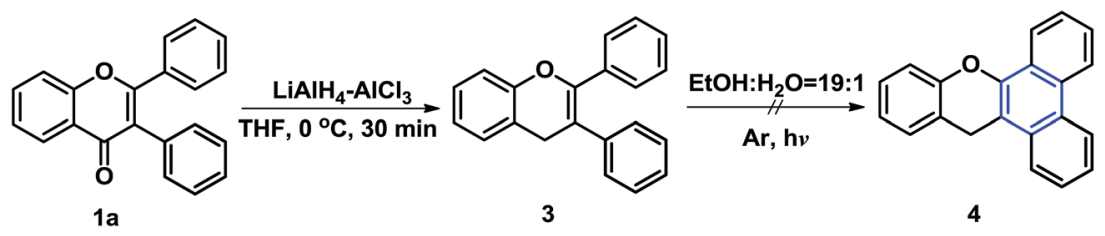

Scheme 3 Reductive carbonyl and attempt annulation of 3. 
126.22, 126.09, 123.73, 118.36, 118.16, 117.27, 116.16, 111.13, 105.19, 77.48, 77.16, 76.84, 55.68, 35.15, 31.60, 21.18; IR (KBr), $\nu$ $\left(\mathrm{cm}^{-1}\right)$ 2941, 1623, 1396, 1259, 1168, 1047, 854, 800, 576; HRMS (ESI): calc. for $\mathrm{C}_{27} \mathrm{H}_{24} \mathrm{O}_{3}[\mathrm{M}+\mathrm{H}]^{+} 397.1804$, found 397.1804.

12-Methyl-14H-dibenzo[a,c] xanthen-14-one (2e). White solid. Yield: $62 \%, M_{\mathrm{p}}$ : 251.9-254.3 ${ }^{\circ} \mathrm{C} .{ }^{1} \mathrm{H}$ NMR $(600 \mathrm{MHz}$, $\left.\mathrm{CDCl}_{3}\right) \delta 10.20(\mathrm{~d}, J=8.3 \mathrm{~Hz}, 1 \mathrm{H}), 8.76(\mathrm{~d}, J=8.3 \mathrm{~Hz}, 1 \mathrm{H}), 8.68$ $(\mathrm{dd}, J=12.8,8.3 \mathrm{~Hz}, 2 \mathrm{H}), 8.23(\mathrm{~s}, 1 \mathrm{H}), 7.84(\mathrm{dd}, J=11.2,4.0 \mathrm{~Hz}$, 1H), 7.78-7.72 (m, 2H), 7.69 (dd, $J=11.2,4.0 \mathrm{~Hz}, 1 \mathrm{H}), 7.60-7.55$ $(\mathrm{m}, 2 \mathrm{H}), 2.53(\mathrm{~s}, 3 \mathrm{H}) ;{ }^{13} \mathrm{C} \mathrm{NMR}\left(100 \mathrm{MHz}, \mathrm{CDCl}_{3}\right) \delta$ 178.51, 155.25, 152.78, 135.17, 134.68, 133.96, 130.63, 129.24, 128.64, 127.88, 127.56, 127.39, 126.70, 126.12, 124.22, 124.18, 123.75, 122.98, 122.42, 117.38, 112.70, 21.21; IR (KBr), $\nu\left(\mathrm{cm}^{-1}\right) 3045$, 2960, 1739, 1629, 1487, 1392, 1271, 1037, 804, 719, 518; HRMS (ESI): calc. for $\mathrm{C}_{22} \mathrm{H}_{14} \mathrm{O}_{2}[\mathrm{M}+\mathrm{H}]^{+} 311.1072$, found 311.1071.

3-(tert-Butyl)-12-methyl-14-H-dibenzo[ $[\boldsymbol{a}, \boldsymbol{c}]$ xanthen-14-one (2f). White solid. Yield: $71 \%, M_{\mathrm{p}}: 208.3-211.1{ }^{\circ} \mathrm{C} .{ }^{1} \mathrm{H}$ NMR $(400$ $\left.\mathrm{MHz} \mathrm{CDCl}_{3}\right) \delta 10.00(\mathrm{~d}, J=8.9 \mathrm{~Hz}, 1 \mathrm{H}), 8.56(\mathrm{~d}, J=8.3 \mathrm{~Hz}, 2 \mathrm{H})$, $8.49(\mathrm{~d}, J=7.7 \mathrm{~Hz}, 1 \mathrm{H}), 8.07$ (s, $1 \mathrm{H}), 7.79(\mathrm{dd}, J=8.9,1.9 \mathrm{~Hz}$, $1 \mathrm{H}), 7.72-7.65(\mathrm{~m}, 1 \mathrm{H}), 7.56(\mathrm{t}, J=7.5 \mathrm{~Hz}, 1 \mathrm{H}), 7.40-7.33(\mathrm{~m}$, 2H), $\left.2.42(\mathrm{~s}, 3 \mathrm{H}), 1.51(\mathrm{~s}, 9 \mathrm{H}) ;{ }^{13} \mathrm{C} \mathrm{NMR} \mathrm{(100} \mathrm{MHz,} \mathrm{CDCl}_{3}\right)$ $\delta$ 178.20, 154.60, 152.56, 149.11, 134.82, 134.29, 133.91, 130.21, 127.52, 127.14, 126.96, 126.77, 126.58, 125.88, 124.05, 123.50, 122.63, 118.10, 117.23, 112.46, 35.14, 31.58, 21.10; IR (KBr), $\nu$ $\left(\mathrm{cm}^{-1}\right)$ 3060, 2952, 1635, 1488, 1398, 1284, 1124, 1033, 923, 806, 763, 636, 522; HRMS (ESI): calc. for $\mathrm{C}_{26} \mathrm{H}_{22} \mathrm{O}_{2}[\mathrm{M}+\mathrm{Na}]^{+}$ 389.1517 , found 389.1514 .

3,12-Dimethyl-14-H-dibenzo[a,c]xanthen-14-one (2g). White solid. Yield: $68 \%, M_{\mathrm{p}}$ : 231.8-233.5 ${ }^{\circ} \mathrm{C} .{ }^{1} \mathrm{H}$ NMR $(400 \mathrm{MHz}$, $\left.\mathrm{CDCl}_{3}\right) \delta 10.07(\mathrm{~d}, J=8.6 \mathrm{~Hz}, 1 \mathrm{H}), 8.75(\mathrm{dd}, J=8.2,1.0 \mathrm{~Hz}, 1 \mathrm{H})$, $8.69(\mathrm{~d}, J=8.3 \mathrm{~Hz}, 1 \mathrm{H}), 8.46(\mathrm{~s}, 1 \mathrm{H}), 8.23(\mathrm{~s}, 1 \mathrm{H}), 7.86-7.80(\mathrm{~m}$, 1H), 7.76-7.70 (m, 1H), 7.62-7.55 (m, 3H), $2.63(\mathrm{~s}, 3 \mathrm{H}), 2.53(\mathrm{~s}$, $3 \mathrm{H}) ;{ }^{13} \mathrm{C}$ NMR $\left(100 \mathrm{MHz}, \mathrm{CDCl}_{3}\right) \delta 178.57,154.69,152.87$, 136.41, 135.13, 134.59, 133.80, 130.49, 130.22, 127.75, 127.72, 127.28, 126.89, 126.13, 124.33, 124.23, 123.76, 123.01, 122.41, 117.41, 112.73, 21.99, 21.22; IR (KBr), $\nu\left(\mathrm{cm}^{-1}\right)$ 2910, 1930, 1610, 1431, 1398, 1286, 1139, 1043, 923, 819, 723, 584, 520; HRMS (ESI): calc. for $\mathrm{C}_{23} \mathrm{H}_{16} \mathrm{O}_{2}[\mathrm{M}+\mathrm{Na}]^{+} 347.1048$, found 347.1052.

6,12-Dimethyl-14-H-dibenzo[a,c] xanthen-14-one (2h). White solid. Yield: $74 \%, M_{\mathrm{p}}$ : 223.7-226.2 ${ }^{\circ} \mathrm{C} .{ }^{1} \mathrm{H}$ NMR $(400 \mathrm{MHz}$, $\left.\mathrm{CDCl}_{3}\right) \delta 10.12(\mathrm{~d}, J=8.3 \mathrm{~Hz}, 1 \mathrm{H}), 8.54(\mathrm{~d}, J=8.1 \mathrm{~Hz}, 1 \mathrm{H}), 8.45$ $(\mathrm{d}, J=8.1 \mathrm{~Hz}, 1 \mathrm{H}), 8.32(\mathrm{~s}, 1 \mathrm{H}), 8.16(\mathrm{~s}, 1 \mathrm{H}), 7.71(\mathrm{t}, J=7.5 \mathrm{~Hz}$, $1 \mathrm{H}), 7.63-7.59(\mathrm{~m}, 1 \mathrm{H}), 7.52-7.40(\mathrm{~m}, 3 \mathrm{H}), 2.58(\mathrm{~s}, 3 \mathrm{H}), 2.50(\mathrm{~s}$, $3 \mathrm{H}) ;{ }^{13} \mathrm{C}$ NMR $\left(101 \mathrm{MHz}, \mathrm{CDCl}_{3}\right) \delta 178.32,155.35,152.63$, $140.95,134.92$, 134.44, 133.93, 129.39, 128.94, 128.40, 127.79, 127.25, 126.41, 126.01, 124.01, 123.68, 122.74, 122.29, 121.83, 117.29, 111.94, 22.42, 21.18; IR (KBr), $\nu\left(\mathrm{cm}^{-1}\right)$ 3028, 2914, 1720, 1610, 1429, 1394, 1282, 1033, 919, 806, 524; HRMS (ESI): calc. for $\mathrm{C}_{23} \mathrm{H}_{16} \mathrm{O}_{2}[\mathrm{M}+\mathrm{H}]^{+}$325.1229, found 325.1224.

3,6,12-Trimethyl-14H-dibenzo[a,c]xanthen-14-one White solid. Yield: $72 \%, M_{\mathrm{p}}: 254.3-256.8{ }^{\circ} \mathrm{C} .{ }^{1} \mathrm{H}$ NMR $(400 \mathrm{MHz}$, $\left.\mathrm{CDCl}_{3}\right) \delta 9.97(\mathrm{~d}, J=8.6 \mathrm{~Hz}, 1 \mathrm{H}), 8.41(\mathrm{~d}, J=8.3 \mathrm{~Hz}, 1 \mathrm{H}), 8.29$ (s, $2 \mathrm{H}), 8.16(\mathrm{~s}, 1 \mathrm{H}), 7.50(\mathrm{dd}, J=11.1,4.7 \mathrm{~Hz}, 2 \mathrm{H}), 7.45$ (d, $J=$ $8.4 \mathrm{~Hz}, 1 \mathrm{H}), 7.39$ (d, $J=8.4 \mathrm{~Hz}, 1 \mathrm{H}), 2.58$ (d, $J=3.9 \mathrm{~Hz}, 6 \mathrm{H}), 2.50$ $(\mathrm{s}, 3 \mathrm{H}) ;{ }^{13} \mathrm{C} \mathrm{NMR}\left(100 \mathrm{MHz}, \mathrm{CDCl}_{3}\right) \delta 178.26,154.83,152.65$, 140.68, 135.97, 134.79, 134.28, 133.69, 129.89, 128.74, 127.62, $127.31,126.98,125.97,123.95,123.64,122.67,122.20,121.89$,
117.28, 111.99, 22.38, 21.92, 21.17; IR (KBr), $\nu\left(\mathrm{cm}^{-1}\right)$ 2914, 1631, 1433, 1402, 1284, 1043, 869, 817, 669, 530; HRMS (ESI): calc. for $\mathrm{C}_{24} \mathrm{H}_{18} \mathrm{O}_{2}[\mathrm{M}+\mathrm{H}]^{+}$339.1385, found 339.1375.

3-(tert-Butyl)-6,12-dimethyl-14H-dibenzo[a,c] xanthen-14-one (2j). Yellow solid. Yield: $78 \%, M_{\mathrm{p}}$ : 260.2-262.8 ${ }^{\circ} \mathrm{C} .{ }^{1} \mathrm{H}$ NMR (400 $\left.\mathrm{MHz} \mathrm{CDCl}_{3}\right) \delta 10.02(\mathrm{~d}, J=8.9 \mathrm{~Hz}, 1 \mathrm{H}), 8.53(\mathrm{~d}, J=1.6 \mathrm{~Hz}, 1 \mathrm{H})$, 8.35 (d, $J=8.3 \mathrm{~Hz}, 1 \mathrm{H}), 8.29$ (s, 1H), 8.11 (s, 1H), 7.80 (dd, $J=$ 8.9, $1.9 \mathrm{~Hz}, 1 \mathrm{H}), 7.43(\mathrm{dd}, J=8.5,1.9 \mathrm{~Hz}, 1 \mathrm{H}), 7.36(\mathrm{dd}, J=8.0$, $4.2 \mathrm{~Hz}, 2 \mathrm{H}), 2.57$ (s, 3H), 2.46 (s, 3H), 1.54 (s, 9H); ${ }^{13} \mathrm{C}$ NMR (100 $\left.\mathrm{MHz}, \mathrm{CDCl}_{3}\right) \delta 178.14,154.87,152.56,148.92,140.63,134.69$, $134.19,134.04,128.67,127.51,127.02,126.93,126.45,125.88$, $123.96,123.55,122.43,121.86,118.04,117.22$, 111.84, 35.15, 31.64, 22.42, 21.11; IR (KBr), $\nu\left(\mathrm{cm}^{-1}\right)$ 3030, 2952, 1629, 1431, 1402, 1284, 1193, 1039, 850, 808, 659, 536; HRMS (ESI): calc. for $\mathrm{C}_{27} \mathrm{H}_{24} \mathrm{O}_{2}[\mathrm{M}+\mathrm{H}]^{+} 381.1855$, found 381.1855.

6-Methyl-14H-dibenzo[a,c]xanthen-14-one (2k). Yellow solid. Yield: $70 \%, M_{\mathrm{p}}$ : $185.7-187.6{ }^{\circ} \mathrm{C} .{ }^{1} \mathrm{H}$ NMR (600 MHz, $\mathrm{CDCl}_{3}$ ) $\delta 10.01(\mathrm{~d}, J=8.3 \mathrm{~Hz}, 1 \mathrm{H}), 8.38(\mathrm{~d}, J=8.1 \mathrm{~Hz}, 1 \mathrm{H}), 8.34(\mathrm{dd}, J=$ 7.8, $1.3 \mathrm{~Hz}, 1 \mathrm{H}), 8.23(\mathrm{~d}, J=8.2 \mathrm{~Hz}, 1 \mathrm{H}), 8.13(\mathrm{~s}, 1 \mathrm{H}), 7.66-7.61$ $(\mathrm{m}, 2 \mathrm{H}), 7.52(\mathrm{dd}, J=11.0,3.9 \mathrm{~Hz}, 1 \mathrm{H}), 7.45(\mathrm{~d}, J=8.2 \mathrm{~Hz}, 1 \mathrm{H})$, $7.38(\mathrm{t}, J=7.4 \mathrm{~Hz}, 1 \mathrm{H}), 7.26(\mathrm{~d}, J=8.2 \mathrm{~Hz}, 1 \mathrm{H}), 2.47(\mathrm{~s}, 3 \mathrm{H}) ;{ }^{13} \mathrm{C}$ NMR (150 MHz, $\left.\mathrm{CDCl}_{3}\right) \delta 178.05,155.16,154.22,140.90,133.77$, 133.53, 129.11, 128.80, 128.27, 127.66, 127.11, 126.58, 126.34, 124.50, 123.93, 123.78, 122.55, 122.17, 121.47, 117.46, 111.82, 22.34; IR (KBr), $\nu\left(\mathrm{cm}^{-1}\right)$ 3055, 2918, 1633, 1407, 1294, 1112, 1024, 873, 757, 520; HRMS (ESI): calc. for $\mathrm{C}_{22} \mathrm{H}_{14} \mathrm{O}_{2}[\mathrm{M}+\mathrm{Na}]^{+}$ 333.0891 , found 333.0893 .

3-(tert-Butyl)-6-methyl-14H-dibenzo[a,c] xanthen-14-one (2l). Yellow solid. Yield: $73 \%, M_{\mathrm{p}}$ : 212.2-214.4 ${ }^{\circ} \mathrm{C}$. ${ }^{1} \mathrm{H}$ NMR $(600 \mathrm{MHz}$, $\left.\mathrm{CDCl}_{3}\right) \delta 10.06(\mathrm{~d}, J=8.8 \mathrm{~Hz}, 1 \mathrm{H}), 8.60(\mathrm{~d}, J=1.8 \mathrm{~Hz}, 1 \mathrm{H}), 8.53$ $(\mathrm{d}, J=8.3 \mathrm{~Hz}, 1 \mathrm{H}), 8.45-8.38(\mathrm{~m}, 2 \mathrm{H}), 7.82(\mathrm{dd}, J=8.8,2.0 \mathrm{~Hz}$, 1H), 7.74-7.69 (m, 1H), $7.60(\mathrm{~d}, J=8.2 \mathrm{~Hz}, 1 \mathrm{H}), 7.48$ (d, $J=$ $8.2 \mathrm{~Hz}, 1 \mathrm{H}), 7.46-7.41(\mathrm{~m}, 1 \mathrm{H}), 2.65(\mathrm{~s}, 3 \mathrm{H}), 1.53(\mathrm{~s}, 9 \mathrm{H}) ;{ }^{13} \mathrm{C}$ NMR $\left(150 \mathrm{MHz}, \mathrm{CDCl}_{3}\right) \delta 178.27,155.14,154.51,149.22,140.94$, 134.31, 133.62, 128.88, 127.57, 127.10, 126.97, 126.73, 126.64, $124.57,124.11,124.07,122.66,121.93,118.17,117.58,112.12$, 35.20, 31.64, 22.51; IR (KBr), $\nu\left(\mathrm{cm}^{-1}\right)$ 3099, 2956, 1635, 1463, 1411, 1296, 1122, 1012, 873, 761, 644, 559, 470; HRMS (ESI): calc. for $\mathrm{C}_{26} \mathrm{H}_{22} \mathrm{O}_{2}[\mathrm{M}+\mathrm{Na}]^{+} 389.1517$, found 389.1516.

2,6-Dimethyl-14H-dibenzo[a,c] xanthen-14-one (2m). Yellow solid. Yield: $75 \%, M_{\mathrm{p}}$ : 218.5-221.1 ${ }^{\circ} \mathrm{C} .{ }^{1} \mathrm{H}$ NMR $(600 \mathrm{MHz}$, $\left.\mathrm{CDCl}_{3}\right) \delta 9.79(\mathrm{~s}, 1 \mathrm{H}), 8.33(\mathrm{~d}, J=7.8 \mathrm{~Hz}, 1 \mathrm{H}), 8.23(\mathrm{t}, J=8.1 \mathrm{~Hz}$, $2 \mathrm{H}), 8.08(\mathrm{~s}, 1 \mathrm{H}), 7.65$ (dd, $J=11.1,4.0 \mathrm{~Hz}, 1 \mathrm{H}), 7.45$ (d, $J=$ $8.1 \mathrm{~Hz}, 1 \mathrm{H}), 7.38(\mathrm{t}, J=7.4 \mathrm{~Hz}, 1 \mathrm{H}), 7.31(\mathrm{~d}, J=8.1 \mathrm{~Hz}, 1 \mathrm{H}), 7.24$ $(\mathrm{d}, J=9.2 \mathrm{~Hz}, 1 \mathrm{H}), 2.54(\mathrm{~s}, 3 \mathrm{H}), 2.46(\mathrm{~s}, 3 \mathrm{H}) ;{ }^{13} \mathrm{C} \mathrm{NMR}(150 \mathrm{MHz}$, $\left.\mathrm{CDCl}_{3}\right) \delta 178.11,155.29,154.21,140.76,138.21,133.86,133.44$, 129.12 , 128.30, 127.81, 127.39, 126.52, 124.87, 124.44, 123.93, 123.75, 122.32, 122.04, 121.05, 117.44, 111.66, 22.34, 22.16; IR $(\mathrm{KBr}), \nu\left(\mathrm{cm}^{-1}\right)$ 3028, 2914, 1637, 1415, 1307, 1172, 1033, 873, 761, 667, 576; HRMS (ESI): calc. for $\mathrm{C}_{23} \mathrm{H}_{16} \mathrm{O}_{2}[\mathrm{M}+\mathrm{Na}]^{+}$ 347.1048 , found 347.1048 .

11-Methoxy-6-methyl-14H-dibenzo[a,c] xanthen-14-one (2n). White solid. Yield: $59 \%, M_{\mathrm{p}}$ : $190.2-191.8{ }^{\circ} \mathrm{C} .{ }^{1} \mathrm{H}$ NMR $(600 \mathrm{MHz}$, $\left.\mathrm{CDCl}_{3}\right) \delta 10.15(\mathrm{~d}, J=8.2 \mathrm{~Hz}, 1 \mathrm{H}), 8.54(\mathrm{~d}, J=8.2 \mathrm{~Hz}, 1 \mathrm{H}), 8.40$ $(\mathrm{d}, J=8.8 \mathrm{~Hz}, 1 \mathrm{H}), 8.32(\mathrm{~s}, 1 \mathrm{H}), 8.27(\mathrm{~d}, J=8.8 \mathrm{~Hz}, 1 \mathrm{H}), 7.70(\mathrm{t}, J$ $=7.4 \mathrm{~Hz}, 1 \mathrm{H}), 7.62-7.60(\mathrm{~m}, 1 \mathrm{H}), 7.41(\mathrm{~d}, J=8.1 \mathrm{~Hz}, 1 \mathrm{H}), 6.96(\mathrm{~d}$, $J=7.4 \mathrm{~Hz}, 1 \mathrm{H}), 6.90(\mathrm{~s}, 1 \mathrm{H}), 3.92(\mathrm{~s}, 3 \mathrm{H}), 2.58(\mathrm{~s}, 3 \mathrm{H}) ;{ }^{13} \mathrm{C} \mathrm{NMR}$ 
$\left(150 \mathrm{MHz}, \mathrm{CDCl}_{3}\right) \delta 177.70,164.23,156.09,155.24,140.79$, 133.78, 129.43, 128.92, 128.33, 128.09, 127.89, 127.31, 126.38, 123.80, 122.72, 122.27, 121.72, 117.99, 114.04, 111.91, 99.62, 55.95, 22.42; IR (KBr), $\nu\left(\mathrm{cm}^{-1}\right)$ 3016, 2918, 1622, 1444, 1249, 1031, 923, 783, 736, 630, 540; HRMS (ESI): calc. for $\mathrm{C}_{23} \mathrm{H}_{16} \mathrm{O}_{3}[\mathrm{M}$ $+\mathrm{Na}]^{+}$341.1178, found 341.1178.

11-Methoxy-2,6-dimethyl-14H-dibenzo[a,c]xanthen-14-one (2o). Yellow solid. Yield: $70 \%, M_{\mathrm{p}}: 221.4-223.8{ }^{\circ} \mathrm{C} .{ }^{1} \mathrm{H}$ NMR $(600 \mathrm{MHz}$, $\left.\mathrm{CDCl}_{3}\right) \delta 9.99(\mathrm{~s}, 1 \mathrm{H}), 8.46(\mathrm{~d}, J=8.3 \mathrm{~Hz}, 2 \mathrm{H}), 8.33(\mathrm{~s}, 1 \mathrm{H}), 8.29(\mathrm{~d}, J$ $=8.8 \mathrm{~Hz}, 1 \mathrm{H}), 7.44(\mathrm{dd}, J=11.3,8.8 \mathrm{~Hz}, 2 \mathrm{H}), 7.01-6.95(\mathrm{~m}, 2 \mathrm{H})$, 3.95 (s, 3H), 2.61 (d, $J=8.9 \mathrm{~Hz}, 6 \mathrm{H}) ;{ }^{13} \mathrm{C} \mathrm{NMR}\left(150 \mathrm{MHz}, \mathrm{CDCl}_{3}\right.$ ) $\delta 177.87,164.25,156.16,155.48,140.77,138.40,133.96,129.50$, 128.52 , 128.09, 127.96, 127.63, 125.14, 123.85, 122.58, 122.21, 121.37, 118.05, 114.06, 111.83, 99.67, 55.98, 22.45, 22.23; IR (KBr), $\nu\left(\mathrm{cm}^{-1}\right)$ 2912, 1627, 1444, 1257, 1172, 1022, 825, 690, 576, 466; HRMS (ESI): calc. for $\mathrm{C}_{24} \mathrm{H}_{18} \mathrm{O}_{3}[\mathrm{M}+\mathrm{Na}]^{+} 377.1154$, found 377.1157 .

3-(tert-Butyl)-11-methoxy-6-methyl-14H-dibenzo[a,c]xanthen14-one (2p). Yellow solid. Yield: $71 \%, M_{\mathrm{p}}$ : 205.6-208.1 ${ }^{\circ} \mathrm{C} .{ }^{1} \mathrm{H}$ NMR (600 MHz, $\left.\mathrm{CDCl}_{3}\right) \delta 10.07(\mathrm{~d}, J=8.8 \mathrm{~Hz}, 1 \mathrm{H}), 8.57$ (d, $J=$ $1.6 \mathrm{~Hz}, 1 \mathrm{H}), 8.40$ (d, $J=8.3 \mathrm{~Hz}, 1 \mathrm{H}), 8.36(\mathrm{~s}, 1 \mathrm{H}), 8.25(\mathrm{~d}, J=$ $8.8 \mathrm{~Hz}, 1 \mathrm{H}), 7.80(\mathrm{dd}, J=8.8,1.9 \mathrm{~Hz}, 1 \mathrm{H}), 7.40(\mathrm{~d}, J=8.3 \mathrm{~Hz}, 1 \mathrm{H})$, $6.92(\mathrm{dd}, J=8.8,2.3 \mathrm{~Hz}, 1 \mathrm{H}), 6.87(\mathrm{~d}, J=2.3 \mathrm{~Hz}, 1 \mathrm{H}), 3.89(\mathrm{~s}, 3 \mathrm{H})$, $2.61(\mathrm{~s}, 3 \mathrm{H}), 1.53(\mathrm{~s}, 9 \mathrm{H}) ;{ }^{13} \mathrm{C}$ NMR $\left(150 \mathrm{MHz}, \mathrm{CDCl}_{3}\right) \delta 177.66$, $164.11,156.10,154.87,148.98,140.56$, 133.98, 128.73, 128.00, 127.60, 127.09, 127.05, 126.47, 123.84, 122.51, 121.84, 118.04, 117.92, 113.92, 111.88, 99.57, 55.90, 35.17, 31.63; IR (KBr), $\nu$ $\left(\mathrm{cm}^{-1}\right)$ 3095, 2952, 1627, 1409, 1255, 1170, 1033, 921, 825, 688, 557, 474; HRMS (ESI): calc. for $\mathrm{C}_{27} \mathrm{H}_{24} \mathrm{O}_{3}[\mathrm{M}+\mathrm{Na}]^{+}$419.1623, found 419.1623 .

3-Fluoro-6-methyl-14H-dibenzo[a,c]xanthen-14-one (2q). Yellow solid. Yield: $55 \%, M_{\mathrm{p}}: 267.4-269.8{ }^{\circ} \mathrm{C} .{ }^{1} \mathrm{H}$ NMR $(400$ $\left.\mathrm{MHz}, \mathrm{CDCl}_{3}\right) \delta 10.23-10.14(\mathrm{~m}, 1 \mathrm{H}), 8.58(\mathrm{~d}, J=8.3 \mathrm{~Hz}, 1 \mathrm{H}), 8.43$ $(\mathrm{d}, J=7.9 \mathrm{~Hz}, 1 \mathrm{H}), 8.28(\mathrm{~s}, 1 \mathrm{H}), 8.20$ (d, $J=10.7 \mathrm{~Hz}, 1 \mathrm{H}), 7.78-$ $7.75(\mathrm{~m}, 1 \mathrm{H}), 7.66$ (d, $J=8.3 \mathrm{~Hz}, 1 \mathrm{H}), 7.55(\mathrm{~d}, J=8.3 \mathrm{~Hz}, 1 \mathrm{H})$, 7.46 (dd, $J=17.2,9.5 \mathrm{~Hz}, 2 \mathrm{H}), 2.65$ (s, 3H); ${ }^{13} \mathrm{C}$ NMR $(150 \mathrm{MHz}$, $\left.\mathrm{CDCl}_{3}\right) \delta 178.16,161.56\left(\mathrm{~d},{ }^{1} J=244.92 \mathrm{~Hz}\right), 155.08,154.56$, 141.44, 133.95, 133.34 (d, $\left.{ }^{4} J=3.87 \mathrm{~Hz}\right), 130.38\left(\mathrm{~d},{ }^{3} J=8.18 \mathrm{~Hz}\right)$, 129.79, 129.58 (d, $\left.{ }^{3} J=7.58 \mathrm{~Hz}\right), 126.82,125.77,124.85,124.29$, 124.04, 123.16, 122.37, 117.65, 116.78 (d, $\left.{ }^{2} J=21.72 \mathrm{~Hz}\right), 112.00$, $107.86\left(\mathrm{~d},{ }^{2} J=22.17 \mathrm{~Hz}\right), 22.46$; IR (KBr), $\nu\left(\mathrm{cm}^{-1}\right) 3060,2918$, 1934, 1635, 1469, 1411, 1226, 1116, 813, 750, 638, 536; HRMS (ESI): calc. for $\mathrm{C}_{22} \mathrm{H}_{13} \mathrm{FO}_{2}[\mathrm{M}+\mathrm{H}]^{+} 329.0978$, found 329.0968 .

3-Chloro-11-methoxy-6-methyl-14H-dibenzo[a,c] xanthen-14one (2r). White solid. Yield: $58 \%, M_{\mathrm{p}}: 237.2-239.1{ }^{\circ} \mathrm{C} .{ }^{1} \mathrm{H}$ NMR $\left(600 \mathrm{MHz}, \mathrm{CDCl}_{3}\right) \delta 10.09(\mathrm{~d}, J=9.0 \mathrm{~Hz}, 1 \mathrm{H}), 8.45(\mathrm{~d}, J=2.0 \mathrm{~Hz}$, $1 \mathrm{H}), 8.42(\mathrm{~d}, J=8.8 \mathrm{~Hz}, 1 \mathrm{H}), 8.26(\mathrm{~d}, J=8.8 \mathrm{~Hz}, 1 \mathrm{H}), 8.22(\mathrm{~s}, 1 \mathrm{H})$, 7.61 (dd, $J=9.0,2.1 \mathrm{~Hz}, 1 \mathrm{H}), 7.47$ (d, $J=8.2 \mathrm{~Hz}, 1 \mathrm{H}), 7.00$ (dd, $J$ $=8.8,2.3 \mathrm{~Hz}, 1 \mathrm{H}), 6.95(\mathrm{~d}, J=2.2 \mathrm{~Hz}, 1 \mathrm{H}), 3.97(\mathrm{~s}, 3 \mathrm{H}), 2.61(\mathrm{~s}$, $3 \mathrm{H}) ;{ }^{13} \mathrm{C}$ NMR $\left(150 \mathrm{MHz}, \mathrm{CDCl}_{3}\right) \delta 177.41,164.46,156.14$, $155.15,141.23$, 132.64, 132.62, 129.61, 129.53, 128.80, 128.50, $128.15,127.72,123.92,122.81,122.13,121.94,117.86,114.25$, 111.54, 99.72, 56.03, 22.41; IR (KBr), $\nu\left(\mathrm{cm}^{-1}\right) 2920,1627,1444$, 1407, 1253, 1164, 1037, 906, 821, 759, 686, 538; HRMS (ESI): calc. for $\mathrm{C}_{23} \mathrm{H}_{15} \mathrm{ClO}_{3}[\mathrm{M}+\mathrm{H}]^{+} 375.0788$, found 375.0783.

3-Fluoro-6-methoxy-12-methyl-14H-dibenzo[a,c]xanthen-14one (2s). White solid. Yield: $64 \%, M_{\mathrm{p}}: 253.4-256.1{ }^{\circ} \mathrm{C} .{ }^{1} \mathrm{H}$ NMR $\left(600 \mathrm{MHz}, \mathrm{CDCl}_{3}\right) \delta 10.16(\mathrm{~s}, 1 \mathrm{H}), 8.52(\mathrm{~d}, J=8.3 \mathrm{~Hz}, 1 \mathrm{H}), 8.16(\mathrm{~s}$, $1 \mathrm{H}), 8.04(\mathrm{~d}, J=8.3 \mathrm{~Hz}, 1 \mathrm{H}), 7.75(\mathrm{~s}, 1 \mathrm{H}), 7.46(\mathrm{dd}, J=31.1$, $24.0 \mathrm{~Hz}, 3 \mathrm{H}), 7.25(\mathrm{~s}, 1 \mathrm{H}), 4.02(\mathrm{~s}, 3 \mathrm{H}), 2.50(\mathrm{~s}, 3 \mathrm{H}) ;{ }^{13} \mathrm{C} \mathrm{NMR}$ $\left(150 \mathrm{MHz}, \mathrm{CDCl}_{3}\right) \delta 177.90,161.41\left(\mathrm{~d},{ }^{1} J=244.71 \mathrm{~Hz}\right), 161.86$, 154.94, 152.79, 135.03, 134.96, 134.58, 130.52 (d, $\left.{ }^{3} J=7.88 \mathrm{~Hz}\right)$, $129.10\left(\mathrm{~d},{ }^{4} J=3.74 \mathrm{~Hz}\right), 126.33,126.16\left(\mathrm{~d},{ }^{4} J=4.86 \mathrm{~Hz}\right), 123.71$, 118.62, 117.69, 117.26, 116.85 (d, $\left.{ }^{2} J=21.81 \mathrm{~Hz}\right), 110.92,107.72$ $\left(\mathrm{d},{ }^{2} J=21.99 \mathrm{~Hz}\right), 104.89,55.73,21.15 ; \mathrm{IR}(\mathrm{KBr}), \nu\left(\mathrm{cm}^{-1}\right) 3105$, 2964, 1917, 1610, 1458, 1234, 1026, 813, 636, 536; HRMS (ESI): calc. for $\mathrm{C}_{23} \mathrm{H}_{15} \mathrm{FO}_{3}[\mathrm{M}+\mathrm{H}]^{+}$359.1083, found 359.1089.

3-Fluoro-11-methoxy-6-methyl-14H-dibenzo[a,c]xanthen-14one (2t). White solid. Yield: $55 \%, M_{\mathrm{p}}: 220.3-222.1{ }^{\circ} \mathrm{C}$. ${ }^{1} \mathrm{H}$ NMR $\left(400 \mathrm{MHz}, \mathrm{CDCl}_{3}\right) \delta 10.21(\mathrm{dd}, J=9.4,6.3 \mathrm{~Hz}, 1 \mathrm{H}), 8.52(\mathrm{~d}, J=$ $8.4 \mathrm{~Hz}, 1 \mathrm{H}), 8.31$ (d, $J=8.5 \mathrm{~Hz}, 1 \mathrm{H}), 8.26(\mathrm{~s}, 1 \mathrm{H}), 8.18(\mathrm{dd}, J=$ 10.9, $2.6 \mathrm{~Hz}, 1 \mathrm{H}), 7.52$ (d, $J=8.4 \mathrm{~Hz}, 1 \mathrm{H}), 7.47-7.40(\mathrm{~m}, 1 \mathrm{H})$, 7.04-6.99 (m, 2H), 3.98 (s, 3H), 2.63 (s, 3H); ${ }^{13} \mathrm{C}$ NMR (100 MHz, $\left.\mathrm{CDCl}_{3}\right) \delta 177.57,164.46,161.86,161.46\left(\mathrm{~d},{ }^{1} J=224.65 \mathrm{~Hz}\right)$, 156.24, 141.07, 133.03 (d, $\left.{ }^{4} J=3.92 \mathrm{~Hz}\right), 130.44\left(\mathrm{~d},{ }^{3} J=8.09 \mathrm{~Hz}\right)$, 129.64, 129.50 (d, $\left.{ }^{3} J=8.03 \mathrm{~Hz}\right), 128.21,125.89\left(\mathrm{~d},{ }^{4} J=1.74 \mathrm{~Hz}\right)$, 124.02, 123.04, 122.29, 117.96, 116.62 (d, $\left.{ }^{2} J=21.78 \mathrm{~Hz}\right), 114.22$, 111.81, 107.71 (d, $\left.{ }^{2} J=22.50 \mathrm{~Hz}\right), 99.76,56.03,22.41$; IR (KBr), $\nu$ $\left(\mathrm{cm}^{-1}\right)$ 2911, 1627, 1448, 1409, 1255, 1109, 950, 819, 538; HRMS (ESI): calc. for $\mathrm{C}_{23} \mathrm{H}_{15} \mathrm{FO}_{3}[\mathrm{M}+\mathrm{H}]^{+}$359.1083, found 359.1083 .

12-Fluoro-2,6-dimethyl-14H-dibenzo[a,c]xanthen-14-one (2u). White solid. Yield: $54 \%, M_{\mathrm{p}}: 222.4-224.1{ }^{\circ} \mathrm{C} .{ }^{1} \mathrm{H}$ NMR $\left(400 \mathrm{MHz}, \mathrm{CDCl}_{3}\right) \delta 9.85(\mathrm{~s}, 1 \mathrm{H}), 8.42(\mathrm{~d}, J=8.4 \mathrm{~Hz}, 2 \mathrm{H}), 8.30$ $(\mathrm{s}, 1 \mathrm{H}), 8.00(\mathrm{dd}, J=8.5,3.1 \mathrm{~Hz}, 1 \mathrm{H}), 7.57(\mathrm{dd}, J=9.1,4.1 \mathrm{~Hz}$, $1 \mathrm{H}), 7.45-7.40(\mathrm{~m}, 3 \mathrm{H}), 2.60(\mathrm{~s}, 6 \mathrm{H}) ;{ }^{13} \mathrm{C}$ NMR $(100 \mathrm{MHz}$, $\left.\mathrm{CDCl}_{3}\right) \delta 177.42,159.45\left(\mathrm{~d},{ }^{1} J=243.59 \mathrm{~Hz}\right), 155.72,150.60$, $141.28,138.64,134.25,129.02,128.64,128.21,127.49$, 125.15, $125.05\left(\mathrm{~d},{ }^{3} J=7.18 \mathrm{~Hz}\right), 123.98,122.63,122.26$, $121.79\left(\mathrm{~d},{ }^{2} J=25.51 \mathrm{~Hz}\right), 121.14,119.53\left(\mathrm{~d},{ }^{3} J=7.89 \mathrm{~Hz}\right)$, $111.46\left(\mathrm{~d},{ }^{2} J=23.85 \mathrm{~Hz}\right), 111.26,22.46,22.21$; IR $(\mathrm{KBr}), \nu$ $\left(\mathrm{cm}^{-1}\right)$ 3080, 2916, 1895, 1635, 1485, 1272, 817, 655, 541; HRMS (ESI): calc. for $\mathrm{C}_{23} \mathrm{H}_{15} \mathrm{FO}_{2}[\mathrm{M}+\mathrm{H}]^{+} 343.1134$, found 343.1134 .

3-(tert-Butyl)-12-fluoro-6-methyl-14H-dibenzo[a,c]xanthen14-one (2v). Yellow solid. Yield: $63 \%, M_{\mathrm{p}}: 240.2-241.8{ }^{\circ} \mathrm{C} .{ }^{1} \mathrm{H}$ NMR (400 MHz, $\left.\mathrm{CDCl}_{3}\right) \delta 9.95(\mathrm{~d}, J=8.9 \mathrm{~Hz}, 1 \mathrm{H}), 8.55(\mathrm{~d}, J=$ $1.5 \mathrm{~Hz}, 1 \mathrm{H}), 8.40$ (d, $J=8.3 \mathrm{~Hz}, 1 \mathrm{H}), 8.35(\mathrm{~s}, 1 \mathrm{H}), 7.97$ (dd, $J=$ 8.5, 3.0 Hz, 1H), $7.80(\mathrm{dd}, J=8.9,1.8 \mathrm{~Hz}, 1 \mathrm{H}), 7.50(\mathrm{dd}, J=9.0$, $4.1 \mathrm{~Hz}, 1 \mathrm{H}), 7.45-7.35(\mathrm{~m}, 2 \mathrm{H}), 2.63(\mathrm{~s}, 3 \mathrm{H}), 1.53(\mathrm{~s}, 9 \mathrm{H}) ;{ }^{13} \mathrm{C}$ NMR (100 MHz, $\left.\mathrm{CDCl}_{3}\right) \delta 177.25,159.35\left(\mathrm{~d},{ }^{1} J=243.61 \mathrm{~Hz}\right)$, $155.15,150.57,149.38,141.13$, 134.31, 128.89, 127.48, 127.10, 126.69, 126.61, $124.94\left(\mathrm{~d},{ }^{3} \mathrm{~J}=7.19 \mathrm{~Hz}\right), 124.01,122.63,121.68$ $\left(\mathrm{d},{ }^{2} J=25.36 \mathrm{~Hz}\right), 121.64,119.49\left(\mathrm{~d},{ }^{3} J=7.87 \mathrm{~Hz}\right), 118.17,111.43$ $\left(\mathrm{d},{ }^{2} J=23.58 \mathrm{~Hz}\right), 111.35,35.21,31.63,22.51 ; \mathrm{IR}(\mathrm{KBr}), \nu\left(\mathrm{cm}^{-1}\right)$ 3045, 2954, 1633, 1450, 1263, 1122, 1033, 813, 777, 543; HRMS (ESI): calc. for $\mathrm{C}_{26} \mathrm{H}_{21} \mathrm{FO}_{2}[\mathrm{M}+\mathrm{Na}]^{+} 407.1423$, found 407.1418.

6-Fluoro-11-methoxy-2-methyl-14H-dibenzo[a,c] xanthen-14one (2w). Yellow solid. Yield: $65 \%, M_{\mathrm{p}}: 254.4-257.2{ }^{\circ} \mathrm{C} .{ }^{1} \mathrm{H} \mathrm{NMR}$ $\left(400 \mathrm{MHz}, \mathrm{CDCl}_{3}\right) \delta 9.97(\mathrm{~s}, 1 \mathrm{H}), 8.61(\mathrm{dd}, J=9.0,5.9 \mathrm{~Hz}, 1 \mathrm{H})$, $8.31(\mathrm{dd}, J=14.5,8.6 \mathrm{~Hz}, 2 \mathrm{H}), 8.16(\mathrm{dd}, J=11.0,2.0 \mathrm{~Hz}, 1 \mathrm{H})$, 7.47 (d, $J=8.1 \mathrm{~Hz}, 1 \mathrm{H}), 7.39-7.33(\mathrm{~m}, 1 \mathrm{H}), 7.01$ (dd, $J=13.4$, $4.6 \mathrm{~Hz}, 2 \mathrm{H}), 3.97$ (s, 3H), $2.62(\mathrm{~s}, 3 \mathrm{H}) ;{ }^{13} \mathrm{C} \mathrm{NMR}\left(100 \mathrm{MHz}, \mathrm{CDCl}_{3}\right)$ $\delta$ 177.68, 164.43, $164.26\left(\mathrm{~d},{ }^{1} J=248.98 \mathrm{~Hz}\right), 156.14,154.84$, $139.41,136.11$ (d, $\left.{ }^{3} J=9.15 \mathrm{~Hz}\right), 128.25,128.19,127.78,126.78$ 
$\left(\mathrm{d},{ }^{3} J=9.37 \mathrm{~Hz}\right), 124.64\left(\mathrm{~d},{ }^{4} J=3.72 \mathrm{~Hz}\right), 122.47,120.21,117.98$, $115.81\left(\mathrm{~d},{ }^{2} \mathrm{~J}=23.56 \mathrm{~Hz}\right), 114.23,111.9537,111.9448,108.21$ (d, $\left.{ }^{2} J=22.61 \mathrm{~Hz}\right), 99.72,56.02,22.25$; IR (KBr), $\nu\left(\mathrm{cm}^{-1}\right)$ 2974, 1897, 1623, 1417, 1271, 1166, 1051, 821, 607, 430; HRMS (ESI): calc. for $\mathrm{C}_{23} \mathrm{H}_{15} \mathrm{FO}_{3}[\mathrm{M}+\mathrm{H}]^{+}$359.1083, found 359.1083.

3-(tert-Butyl)-6-fluoro-11-methoxy-14H-dibenzo[ $[a, c]$ xanthen14-one (2x). White solid. Yield: $69 \%, M_{\mathrm{p}}: 254.2-256.7{ }^{\circ} \mathrm{C} .{ }^{1} \mathrm{H}$ NMR (400 MHz, $\mathrm{CDCl}_{3}$ ) $\delta 10.01(\mathrm{~d}, J=8.9 \mathrm{~Hz}, 1 \mathrm{H}), 8.47$ (dd, $J=$ $8.9,6.0 \mathrm{~Hz}, 1 \mathrm{H}), 8.38(\mathrm{~s}, 1 \mathrm{H}), 8.22(\mathrm{~d}, J=8.9 \mathrm{~Hz}, 1 \mathrm{H}), 8.18-8.11$ $(\mathrm{m}, 1 \mathrm{H}), 7.85-7.77(\mathrm{~m}, 1 \mathrm{H}), 7.26(\mathrm{~d}, J=6.6 \mathrm{~Hz}, 1 \mathrm{H}), 6.92(\mathrm{dd}, J=$ 8.9, $2.0 \mathrm{~Hz}, 1 \mathrm{H}), 6.83(\mathrm{~s}, 1 \mathrm{H}), 3.90(\mathrm{~s}, 3 \mathrm{H}), 1.51(\mathrm{~s}, 9 \mathrm{H}) ;{ }^{13} \mathrm{C} \mathrm{NMR}$ $\left(100 \mathrm{MHz}, \mathrm{CDCl}_{3}\right) \delta 177.43,164.26,164.03\left(\mathrm{~d},{ }^{1} J=248.80 \mathrm{~Hz}\right)$, 156.04, 154.14, 149.38, 136.03 (d, ${ }^{3} \mathrm{~J}=8.78 \mathrm{~Hz}$ ), 128.04, 127.72, 127.31, 126.66 (d, $\left.{ }^{3} J=9.28 \mathrm{~Hz}\right), 126.48$ (d, $\left.{ }^{4} J=3.55 \mathrm{~Hz}\right), 120.56$, 118.26, 117.79, 115.89 (d, $\left.{ }^{2} J=23.63 \mathrm{~Hz}\right), 114.11,111.95,108.18$ $\left(\mathrm{d},{ }^{2} J=22.75 \mathrm{~Hz}\right), 99.56,55.94,35.18,31.56 ; \mathrm{IR}(\mathrm{KBr}), \nu\left(\mathrm{cm}^{-1}\right)$ 3099, 2960, 1625, 1442, 1255, 1166, 1035, 941, 827, 734, 578, 478; HRMS (ESI): calc. for $\mathrm{C}_{26} \mathrm{H}_{21} \mathrm{FO}_{3}[\mathrm{M}+\mathrm{H}]^{+} 401.1553$, found 401.1562 .

3-(tert-Butyl)-11-hydroxy-6-methyl-14H-dibenzo[a,c]xanthen14-one (2y). Red solid. Yield: 78\%, $M_{\mathrm{p}}$ : 291.6-293.4 ${ }^{\circ} \mathrm{C} .{ }^{1} \mathrm{H}$ NMR $(600 \mathrm{MHz}, \mathrm{DMSO}) \delta 10.75(\mathrm{~s}, 1 \mathrm{H}), 9.83(\mathrm{~d}, J=8.1 \mathrm{~Hz}, 1 \mathrm{H}), 8.56(\mathrm{~s}$, $2 \mathrm{H}), 8.43(\mathrm{~d}, J=8.2 \mathrm{~Hz}, 1 \mathrm{H}), 7.98(\mathrm{~d}, J=8.6 \mathrm{~Hz}, 1 \mathrm{H}), 7.66(\mathrm{~d}, J=$ $8.8 \mathrm{~Hz}, 1 \mathrm{H}), 7.47$ (d, $J=8.1 \mathrm{~Hz}, 1 \mathrm{H}), 6.97$ (s, 1H), 6.85 (d, $J=$ $8.6 \mathrm{~Hz}, 1 \mathrm{H}), 2.37$ (s, 3H), $1.34(\mathrm{~s}, 9 \mathrm{H}) ;{ }^{13} \mathrm{C} \mathrm{NMR}(150 \mathrm{MHz}$, DMSO) $\delta 176.37,162.97,155.74,154.08,148.71,140.91,133.12$, 129.11, 127.64, 126.69, 126.56, 126.34, 125.96, 123.59, 122.80, $121.10,118.47,116.05,114.70,110.60,101.83,34.78,31.22$, 21.67; IR (KBr), $\nu\left(\mathrm{cm}^{-1}\right)$ 3189, 2958, 1620, 1415, 1269, 1174, 923, 821, 559, 468; HRMS (ESI): calc. for $\mathrm{C}_{26} \mathrm{H}_{22} \mathrm{O}_{3}[\mathrm{M}+\mathrm{Na}]^{+}$ 405.1467, found 405.1463.

6-Hydroxy-12-methyl-14H-dibenzo[ $[a, c]$ xanthen-14-one (2z). White solid. Yield: $70 \%, M_{\mathrm{p}}: 299.4-301.2{ }^{\circ} \mathrm{C} .{ }^{1} \mathrm{H}$ NMR $(600 \mathrm{MHz}$, DMSO) $\delta 10.62$ (s, 1H), $10.04(\mathrm{~d}, J=8.3 \mathrm{~Hz}, 1 \mathrm{H}), 8.63-8.59(\mathrm{~m}$, $2 \mathrm{H}), 8.07(\mathrm{~d}, J=1.8 \mathrm{~Hz}, 1 \mathrm{H}), 8.01(\mathrm{~s}, 1 \mathrm{H}), 7.74-7.70(\mathrm{~m}, 2 \mathrm{H})$, 7.68-7.63 (m, 2H), $7.33(\mathrm{dd}, J=8.9,2.1 \mathrm{~Hz}, 1 \mathrm{H}), 2.46(\mathrm{~s}, 3 \mathrm{H}) ;{ }^{13} \mathrm{C}$ NMR (150 MHz, DMSO) $\delta 176.84,160.51,155.20,152.07,135.61$, $135.20,134.28,128.92,128.36,126.81,126.44,126.40,126.20$, $125.03,122.80,122.79,118.15,117.55,116.00,109.31,107.12$, 20.55; IR (KBr), $\nu\left(\mathrm{cm}^{-1}\right) 3145,2912,2314,1608,1436,1244,806$, 619, 526; HRMS (ESI): calc. for $\mathrm{C}_{22} \mathrm{H}_{14} \mathrm{O}_{3}[\mathrm{M}+\mathrm{Na}]^{+}$349.0841, found 349.0839 .

\section{Conflicts of interest}

The authors declare no competing financial interest.

\section{Acknowledgements}

We are grateful for financial support from the National Natural Science Foundation of China (No. 21672132, 21502110).

\section{References}

1 M. Fagnoni, D. Dondi, D. Ravelli and A. Albini, Chem. Rev., 2007, 107, 2725-2756.

2 V. Dichiarante, M. Fagnoni and A. Albini, Green Chem., 2009, 11, 942-945.

3 M. Fagnoni and A. Albini, Acc. Chem. Res., 2005, 38, 713-721. 4 Y. Inoue, Chem. Rev., 1992, 92, 741-770.

5 K. B. Jørgensen, Molecules, 2010, 15, 4334-4358.

6 F. B. Mallory, K. E. Butler, A. Bérubé, E. D. Luzik Jr, C. W. Mallory, E. J. Brondyke, R. Hiremath, P. Ngo and P. J. Carroll, Tetrahedron, 2001, 57, 3715-3724.

7 A. Gaspar, M. J. Matos, J. Garrido, E. Uriarte and F. Borges, Chem. Rev., 2014, 114, 4960-4992.

8 S. Quideau, Angew. Chem., Int. Ed., 2006, 45, 6786-6787.

9 R. S. Keri, S. Budagumpi, R. K. Pai and R. G. Balakrishna, Eur. J. Med. Chem., 2014, 78, 340-374.

10 J. Yang and N. Yoshikai, Angew. Chem., Int. Ed., 2016, 55, 2870-2874.

11 M. C. Jiménez, M. A. Miranda and R. Tormos, Heterocycl. Chem., 1996, 43, 339-347.

12 F. B. Mallory, C. S. Wood and J. T. Gordon, J. Am. Chem. Soc., 1964, 86, 3094-3102.

13 C. S. Wood and F. B. Mallory, J. Org. Chem., 1964, 29, 33733377.

14 A. G. Neo, C. López, V. Romero, B. Antelo, J. Delamano, A. Pérez, D. Fernández, J. F. Almeida, L. Castedo and G. Tojo, J. Org. Chem., 2010, 75, 6764-6770.

15 J. Han, T. Wang, Y. Liang, Y. Li, C. Li, R. Wang, S. Feng and Z. Zhang, Org. Lett., 2017, 19, 3552-3555.

16 Q. Yang, T. Wei, Y. He, Y. Liang and Z.-T. Zhang, Helv. Chim. Acta, 2015, 98, 953-960.

17 Q. Wang, Z. Zhang, Z. Du, H. Hua and S. Chen, Green Chem., 2013, 15, 1048-1054.

18 W. Wei, C. Li, T. Wang, D. Liu and Z. Zhang, Tetrahedron, 2016, 72, 5037-5046.

19 A. G. Lvov, V. Z. Shirinian, A. V. Zakharov, M. M. Krayushkin, V. V. Kachala and I. V. Zavarzin, J. Org. Chem., 2015, 80, 11491-11500.

20 R. Hoffmann and R. B. Woodward, Acc. Chem. Res., 1968, 1, 17-22.

21 A. G. Schultz and I. C. Chiu, J. Chem. Soc., Chem. Commun., 1978, 29.

22 A. G. Schultz, W. Y. Fu, R. D. Lucci, B. G. Kurr, K. M. Lo and M. Boxer, J. Am. Chem. Soc., 1978, 100, 2140-2149.

23 A. G. Schultz, W. Y. Fu, R. D. Lucci, B. G. Kurr, K. M. Lo and M. Boxer, J. Am. Chem. Soc., 1978, 100, 2150-2162.

24 R. Sekiya and R. Kuroda, Chem. Commun., 2011, 47, 1009710099.

25 C. Valla, A. Baeza, F. Menges and A. Pfaltz, Synlett, 2008, 3167-3171.

26 F. J. Zhang and Y. L. Li, Synthesis, 1993, 565-567. 